Bio-grafia: Escritos sobre la Biologia y su Enseñanza Vol 3 No4 ISSN 2027-1034. Primer semestre de 2010, Bogotá, Colombia, pp 191-196

\title{
LA FORMULACIÓN DE PREGUNTAS: UNA ALTERNATIVA METODOLÓGICA QUE FAVORECE LA REFLEXIÓN, DISCUSIÓN Y CONSTRUCCIÓN DE CONOCIMIENTO.
}

Cambios morfológicos en la Mariposa Blanca de col, Leptophobia aripa tras la modificación de su dieta alimenticia en etapas específicas de su desarrollo.

1Por: Natalia Sánchez Media

El Área de Ciencias Naturales y Educaci ón Ambiental del Instituto Pedagógico Arturo Ramírez Montúfar (IPARM) de la Universidad Nacional de Colombia Sede Bogotá, corrobora la importancia de generar metodologías y alternativas que contribuyan al desarrollo en los estudiantes de habilidades científicas básicas que les permitan construir explicaciones a los eventos de orden biológico con una mirada propositiva y crítica, basada en el fomento de valores hacia el cuidado y respeto por su ambiente y las otras especies.

En este sentido, desde del año 2007, y teniendo como antecedente inmediato las investigaciones de campo y laboratorio desarrolladas por la Línea de Investigación Faunística y Conservación de Artrópodos de la Universidad Pedagógica Nacional, se vienen desarrollando un conjunto de actividades cuyo objetivo principal es fortalecer en los estudiantes el conocimiento biológico de algunas especies de invertebrados como vínculo inmediato para la conservación y preservación de otras formas de vida.

La trascendencia de estas iniciativas se evidencia en nociones de respeto, tolerancia y responsabilidad que demuestra los estudiantes entre sí, pues se identifican como una especie más, que al igual que la estudiada, requiere de condiciones adecuadas para su desarrollo.

Las aproximaciones y resultados de estas iniciativas se han divulgado a través de revistas y encuentros con el ánimo de socializar y retroalimentar su sentido y proyección institucional. Entre estos se encuentran: artículo publicado en el cuarto número de la revista Pedagogía en Escena año 2008², ponencia presentada en el Primer Encuentro Nacional de Experiencias de Enseñanza de la Biología y la

\footnotetext{
${ }^{1}$ Natalia Sánchez Media Licenciada en Biología. Universidad Pedagógica Nacional. Docente Ciencias Naturales, Colegio Champagnat, Bogotá.

Correo electrónico: natissame20@hotmail.com
}

2 SANCHEZ, Natalia. 2008. Pedagogía en Escena: En la búsqueda del conocimiento compartido: La adopción de una mariposa como estrategia para el reconocimiento y valoración de otras especies. "El extraordinario mundo de las mariposas". Pg. 22-24. 
Educación Ambiental ${ }^{3}$, artículo publicado en el primer número de la revista electrónica bio - grafía del departamento de biología de la Universidad Pedagógica Nacional ${ }^{4}$ y la participación en la décima primera versión de Expociencia Infantil y Juvenil 2009.

Durante el 2008, con estudiantes de sexto grado del IPARM y, en complemento con lo propuesto en el plan de área para ese nivel, se desarrolló una aproximación a la biología y ecología de las mariposas a través del seguimiento, adopción y registro del ciclo de vida de la mariposa blanca de la col Leptophobia aripa.

Para ello, y como herramienta metodológica fundamental, se extrapolaron algunos principios fundamentales del ciclo de indagación de primera mano (Arango 2002) elemento orientador de la propuesta "La Enseñanza de la Ecología en el Patio de la Escuela (EEPE).

El ciclo de indagación consiste en el desarrollo, seguimiento y la secuencia de una serie de actividades o procedimientos que tienen como objetivo fundamental hacer "investigación" en el contexto del patio de la escuela. El primer paso o procedimiento consiste en la construcción de una o varias preguntas por parte del estudiante. Esta pregunta se origina de su curiosidad la cual es "activada" a partir de la observación de los elementos que le resultan interesantes, llamativos o novedosos de su entorno inmediato y su vinculación con los conocimientos y experiencias relacionadas y que ha aprendido a lo largo de su vida.

Un segundo paso o etapa, se denomina "experiencia de primera mano" o "acción". Consiste en la recolección y análisis de la información que resulta relevante para la resolución de los interrogantes formulados en la fase anterior. Dichas incógnitas se resuelven mediante las acciones que desarrollan los estudiantes, con la ayuda de las herramientas permanentes con las que dispone.

Una última etapa consiste en una "reflexión" sobre la o las respuesta que se obtienen después de desarrolladas las fases anteriores. Este procedimiento incluye una sesión de análisis, la cual debe hacerse con el ánimo de determinar si la respuesta construida es aplicable en un ámbito más amplio que el del Patio de la Escuela. (Arango, 2002).

Durante el proceso de observación, registro y análisis de los cambios experimentados por el organismo (orugas) con el paso del tiempo, los estudiantes reconocieron la estrecha relación que establecen las mariposas con las plantas, las condiciones ideales para su

${ }^{3}$ SANCHEZ, Natalia y TORRES, Nubia. "SEMILLAS AMBIENTALES una propuesta pedagógica de Educación Ambiental en el Instituto Pedagógico Arturo Ramírez Montúfar (IPARM) de la Universidad Nacional de Colombia - Sede Bogotá.

${ }^{4}$ SANCHEZ, Natalia y TORRES, Nubia. Bio-grafia: Escritos sobre la Biología y su Enseñanza: "Semillas Ambientales" en el Instituto Pedagógico Arturo Ramírez Montúfar (IPARM) de la Universidad Nacional de Colombia - Sede Bogotá. 
desarrollo y crecimiento, así como la importancia de estos fascinantes organismos en la regulación y mantenimiento de los ecosistemas.

Una de los elementos más relevantes, y que lograron identificar y analizar, es la importancia de seleccionar adecuadamente la planta que servirá de alimento a los estados inmaduros de las mariposas.

Las mariposas son a menudo muy selectivas con respecto a su alimentación mientras se encuentran como orugas y usualmente mueren antes de comer una planta que no es su hospedera o nutricia (Torres y García, 2004). Teniendo en cuenta esta particularidad, todos los estudiantes alimentaron sus orugas (estados inmaduros de L. aripa) con una enredadera que hace parte de la familia Tropaeolaceae. Se conoce comúnmente como "capuchina" por la característica forma de su hoja.

Esta peculiar mariposa, sin embargo, se hospeda adicionalmente en plantas que hacen parte de la familia Brassicaceae. Dentro de esta familia se encuentran un gran número de plantas que se cultivan y usan para alimentación de poblaciones humanas entre las más conocidas están quizás el repollo, coliflor, repollo y brócoli.

La particularidad alimenticia de esta mariposa (consumo de los estados inmaduros de más de una familia de plantas) permitió desarrollar el presente trabajo de indagación el cual giró en torno a una pregunta formulada por el mismo grupo de estudiantes, que durante el año inmediatamente anterior, registraron el ciclo de vida de Leptophobia aripa y es ¿la dieta cambia la apariencia de las mariposas?

De este interrogante surgieron además, otras inquietudes, las cuales orientaron procesos fundamentales que darían lugar a una posible respuesta y la corroboración de las hipótesis formuladas por ellos al inicio del proceso. Entre éstas se encuentran ¿En cualquier etapa puedo modificar o cambiar la alimentación de mi oruga, no importan si es grande o pequeña?; ¿Puedo cambiar su alimentación independiente a la planta en la cual encontré los huevos?; ¿La posibilidad de supervivencia de mi mariposa es igual independiente a la planta que le suministre como alimentación?

A partir de estas inquietudes, los estudiantes del séptimo grado establecieron las pautas metodológicas que les permitiría construir una aproximación o posible resolución a sus inquietudes. Cada estudiante recibió tres orugas con aproximadamente dos días de nacimiento. Los huevos empleados en esta experiencia fueron colectados sobre "capuchina" y la variación o modificación en su dieta se realizó luego de transcurrido este espacio de tiempo. 
Un grupo de estudiantes alimentó y mantuvo su oruga en la misma planta en la cual se colectaron o encontraron los huevos, mientras que el otro equipo de trabajo suministró a su larva, hasta el momento en que formara la crisálida, una variedad de col.

Los parámetros de comparación que tuvieron en cuenta, y que así mismo, les brindó elementos para su discusión fueron: duración aproximada del ciclo de vida, es decir, el tiempo que tardó el organismo en alcanzar sus cuatro etapas de desarrollo (huevo, larva, crisálida y adulto), la coloración de las alas tras la observación detenida de las mariposas adultas y su tamaño.

Durante el proceso, surgieron nuevos interrogantes, todos vinculados con la manipulación y observación detallada de su organismo, entre estas se resalta ¿Por qué la cantidad de alimento consumido por mi oruga varía de un grupo a otro?; ¿Por qué la coloración de mi oruga cambia, y algunas pautas morfológicas no son tan evidentes?; ¿A qué se debe que la cantidad de pelotillas fecales sea diferente en cantidad y aspecto?

Dentro de los resultados de la experiencia, y teniendo en cuenta las preguntas generadas por los estudiantes se encontró que las mariposas a las que se les suministró col emergieron de su crisálida a los 23 días y la coloración de sus alas fue más clara, mientras que el conjunto de larvas que se les suministró "capuchina" completaron su ciclo cuatro días después, es decir a los 27 días. Así mismo, el índice de mortalidad no fue significativamente más alto en ninguno de los dos grupos y a pesar, de que no se diseñó un experimento puntal para evaluarlo, la etapa en la cual fueron realizadas las variaciones en la dieta fue el óptimo. Lo anterior teniendo en cuenta que trascurrido demasiado tiempo el organismo no es capaz de adaptarse favorablemente al cambio en la alimentación (aproximadamente dos días posteriores a la eclosión).

La coloración y tamaño de las larvas, igualmente, varío significativamente, siendo más grandes aquellas alimentadas con capuchina a quienes además las franjas laterales de color amarillo, negras y azules se resaltaron considerablemente sobre todo en las últimas etapas de la fase larvaria.

Durante el desarrollo de la presente propuesta las preguntas e inquietudes formuladas por los estudiantes en cada una de las fases del ciclo de indagación de primera mano fueron un elemento orientador de los procedimientos y métodos puestos en práctica por los mismos en la construcción de una respuesta. En este sentido, se logró fortalecer el desarrollo de habilidades científicas fundamentales entre las que se incluyen: la observación rigurosa de procesos (crecimiento y desarrollo del organismo), la toma de evidencias necesarias en forma gráfica, de tablas y fotografías, la manipulación de variables, la discusión como un espacio de encuentro, intercambio y retroalimentación de ideas a partir de los resultados obtenidos en cada uno de los grupos de trabajo y la 
reflexión en torno a la importancia de conservar los hábitats en los cuales se desarrollan la mariposa blanca de la col Leptophobia aripa.

Se debe resaltar el interés con el cual los estudiantes trabajaron en el laboratorio, desarrollaron la revisión bibliográfica necesaria para orientar sus preguntas y pautas metodológicas y la satisfacción con la cual se aproximaron a sus respuestas. En este sentido, concluyeron que existe una estrecha relación entre el tipo de alimento que se le suministra a una oruga con las características morfológicas del individuo esto como resultado de la cantidad y tipo de pigmentos que contienen las dos familias de plantas trabajadas. Igualmente, llegaron a la conclusión que es posible realizar las variaciones en la alimentación de las larvas independiente a la planta en la cual fueron colectados los huevos, sin embargo, el éxito de que los organismos alcancen la etapa adulta aumenta cuando la modificación se realiza en las primeras etapas larvarias. Lo anterior, producto de un proceso de adaptación a las sustancias que contienen cada una de las plantas.

Reflexionaron además acerca de la posibilidad de realizar la experiencia con otras mariposas, sin embargo, resaltan la importancia de desarrollar con rigurosidad la revisión bibliográfica del organismo antes de proceder a su manipulación y estudio en laboratorio. Entre los parámetros que consideran importantes se encuentran: saber el tipo de planta nutricia u hospedera, algunas características de cada fase o etapa de desarrollo y las condiciones atmosféricas (humedad y temperatura) en la cual se desarrollan de manera adecuada.

La experiencia presentada se convierte en una alternativa concreta en la cual se logran desarrollar procesos de pensamiento vinculados con la formulación de preguntas como elemento orientador, la reflexión y extrapolación de prácticas puntuales llevadas a cabo en el laboratorio con el análisis de las condiciones que requiere un organismo para desarrollarse en su ambiente natural, la importancia de conservar todas las especies de plantas por la relación que tienen con las mariposas, construir conocimiento colectivo a través del fortalecimiento del trabajo en equipo y la manipulación y cercanía con otras formas de vida.

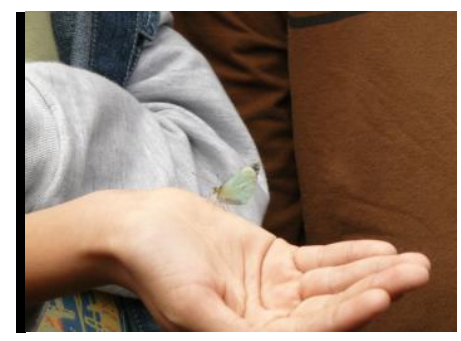

Mariposa adulta. Leptophobia aripa alimentada con Tropaeolum majus ("capuchina"). Fotografía tomada por: Natalia Sánchez Medina

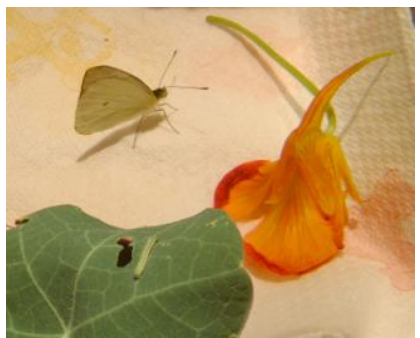

Oruga alimentándose de "Capuchina" y adulto tras atravesar el proceso de la metamorfosis. Fotografía tomada por: Natalia Sánchez Medina

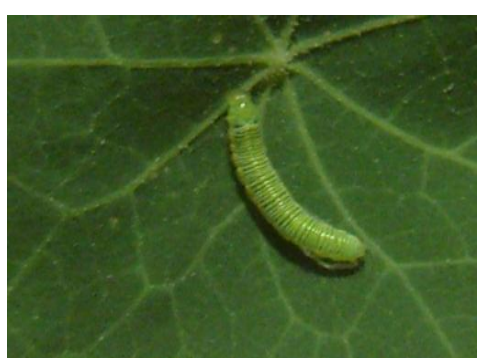

Larva sobre "Capuchina", Tropaeolum majus. Fotografía tomada por: Natalia Sánchez Medina

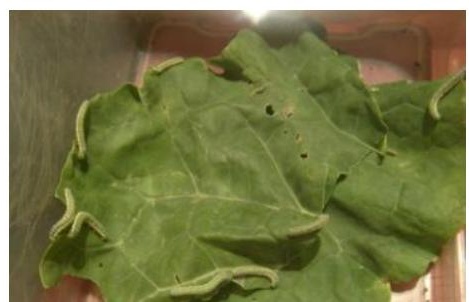


Larva de Leptophobia aripa sobre una variedad de col. Fotografía tomada por: BIE
Crisálida Mariposa Blanca de la Col, Leptophobia aripa. Oruga alimentada con una variedad de col. Fotografía tomada por: Natalia Sánchez Medina

1. ARANGO, Natalia (2002). Guía metodológica para la enseñanza de ecología en el patio de la escuela. Nueva York NY: Audubon, Programa para América Latina y el Caribe.

2. CENTRO AGRONÓMICO TROPICAL DE INVESTIGACIÓN Y ENSEÑANZA. 1990. Guía para el manejo integrado de plagas del cultivo de repollo. Programa de mejoramiento de cultivos tropicales. Turrialba, Costa Rica.

3. LASTRA, José, et al. 2006. Host selection behavior of Leptophobia aripa (lepidóptera: pieridae). Florida Entomologist 89(2).

4. SÁNCHEZ, Raquel. 2004. Protocolo de cría para dos especies de mariposas, Ascia monuste y Leptophobia aripa (Lepidóptera: Pieridae) bajo condiciones controladas en el municipio de la mesa, Cundinamarca. Departamento de Biología. Pontificia Universidad Javeriana.

5. Torres, R. (2004). Proyecto Mariposas para Educar. Línea de Investigación Faunística y Conservación de Artrópodos. Universidad Pedagógica Nacional. 